\title{
UDC 624.042
}

SCOPUS CODE 2205

https://doi.org/10.36073/1512-0996-2020-2-104-109

\section{О реализации одной модели вертикального сейсмического воздействия}

\author{
Серго Эсадзе \\ Департамент промышленного и гражданского строительства им. Агули Сохадзе, \\ Грузинский технический университет, Грузия, 0160, Тбилиси, ул. М. Костава $68^{6}$ \\ E-mail: s_esadze@gtu.ge \\ Тамар Эсадзе \\ Департамент промышленного и гражданского строительства им. Агули Сохадзе, \\ Грузинский технический университет, Грузия, 0160, Тбилиси, ул. М. Костава $68^{6}$ \\ E-mail: t.esadze@gtu.ge \\ Торнике Мамаладзе Департамент промышленного и гражданского строительства им. Агули Сохадзе, \\ Грузинский технический университет, Грузия, 0160, Тбилиси, ул. М. Костава $68^{6}$ \\ E-mail: tornike.mamaladze1994@gmail.com
}

\section{Рецензенты:}

Л. Кахиани, профессор строительного факультета ГТУ

E-mail: L.kakhiani@gtu.ge

Д. Табатадзе, профессор строительного факультета ГТУ

E-mail: Demuritabatadze@gmail.com

Аннотация. В статье рассматриваются вертикальные сейсмические колебания консольных конструкций, которые являются частями рамного каркаса и имеют большой вылет. По всем существующим нормам по сейсмостойкости консоли должны быть рассчитаны на вертикальную сейсмическую нагрузку. В статье даётся реализация метода предложенного авторами, предусматривающего нормативный расчёт консоли на вертикальную сейсмическую нагрузку с учётом влияния статически нагруженных сопредельных конструкций. Рассмотрено реализация метода для двух значений соотношений характеризующих параметров предложенной расчётной модели - жёсткости сопредельной колонны и жёсткости введённого элемента, создающего начальное нагружение в колонне. Полученные значения расчётных сейсмических нагрузок рассмотрены в сравнении с нормативным значением этой же нагрузки, полученной без учёта особенностей предложенной модели.

Ключевые слова: консоль; вертикальное ускорение; сейсмическая нагрузка.

\section{Введение}

Разрушительное влияние вертикального составляющего сейсмического ускорения основания на конструктивную систему и/или элементы зданий и 
сооружений, особенно в эпиценттральной зоне землетрясения подтверждается последствиями сильных эемлетрясений. Наиболее наглядный пример последних, землетрясения Нортридж в 1994 году в США и Кобе в 1995 году в Японии. Отмеченным обьясняются требования норм всех стран расположенных в сейсмоактивных регионах $[1,2,3,4]$, включая Еврокод [5], об учёте вертикального сейсмического ускорения для определённых конструктивных элементов зданий и сооружений. Одним из таких элементов являются горизонтальные и/или наклонные консоли для создания расчётных моделей которых предложены те же принципы, что и для расчёта на горизонтальные ускорения - стержневая система с сосредоточенными массами.

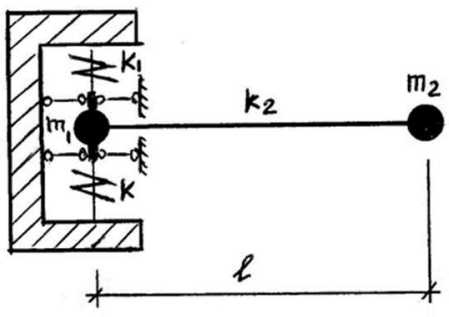

Рис. 1. Расчётная модель

\section{Основная часть}

В работе [6] дана расчётная модель для определения нормативной вертикальной нагрузки действующей на консоли большого вылета (5м и более) плоской рамы являющегося несущей частью рамного каркаса. Модель основана на положении [6], допускающем принятие в виде расчётной модели рассматриваемого конструктивного элемента и сопредельных конструктивных элементов, влияющих на его работу. С учётом всей совокупности особенностей свойственной рассматриваемой нами системе, расчётная модель (рис. 1) характеризуется совокупностью следующих параметров: $k$ - жёсткость сопредельной несущей колонны, $k_{1}$ - коэффициент, учитывающий предварительное статическое нагружение колонны, $k_{2}{ }^{-}$жёсткость самой колонны. Для динамических характеристик системы имеем:
- для частот

$$
\begin{gathered}
\omega_{1,2}^{2}= \\
=\frac{1}{2}\left[\left(\frac{k_{2}}{m_{2}}+\frac{k+k_{1}+k_{2}}{m_{1}}\right) \pm \sqrt{\left(\frac{k_{2}}{m_{2}}+\frac{k+k_{1}+k_{2}}{m_{1}}\right)^{2}-\frac{4 k_{2}\left(k+k_{1}\right)}{m_{1} m_{2}}}\right]
\end{gathered}
$$

- для форм колебаний

$$
\begin{gathered}
\left(m_{1} \omega^{2}-k-k_{1}-k_{2}\right) A_{11}+K_{2} A_{21}=0 \\
K_{2} A_{12}+\left(m_{2} \omega^{2}-K_{2}\right) A_{22}=0
\end{gathered}
$$

Для реализации данной модели, т.е. для определения вертикальной сейсмической нагрузки на консоли по нормативной методике, но с учётом предложенной нами модели, определяющими являются соотношения перечисленных выше параметров - жёсткостей и масс. Ниже даны несколько этих реальных соотношений и соответствующие им нормативные сейсмические нагрузки в сравнении с той же нагрузкой, определённой по нормативной методике, только без учёта особенностей предложенной модели.

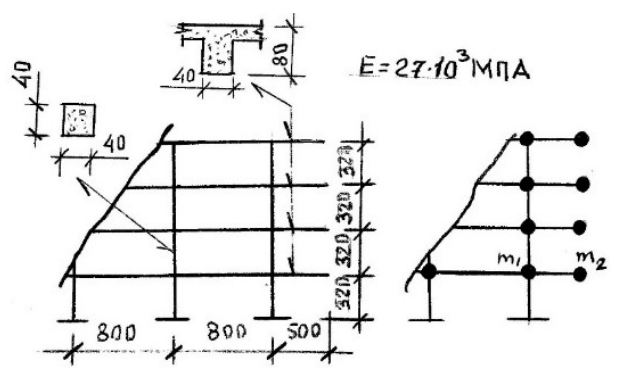

Рис. 2. Данные для расчёта

На рисунке 2 фрагмент рамы с консолями с большим вылетом (5м) с геометрическими и инерционными показателями. Чтобы при начальном возмущении системы появилось продольное усилие в сопредельной к консоли колонне, а система колебалась не симметрично относительно горизонтальной оси, значение должно быть в пределах $0<k_{1}<k$ вместе с этим значения $k_{1}$ берём в пределах $\left(k / k_{1}\right)=$ $1,25 \ldots 1,60$

$\left(k / k_{1}\right)=1,25, m_{1}=23,12 \cdot 10^{3} \kappa г, m_{2}=21,1$. $10^{3}$ кг Для динамических характеристик системы будем иметь (таблица 1 ): 
Таблица 1

\begin{tabular}{|c|c|c|c|c|c|c|c|c|c|c|}
\hline H/M & н/м & н/м & рад/с & с & рад/с & с & & & & \\
\hline 1,35 & 1,08 & 0,006 & 16,8 & 0,4 & 324,6 & 0,02 & 1 & 404,9 & 1 & $-0,0027$ \\
\hline
\end{tabular}

$\left(k / k_{1}\right)=1,6, m_{1}=23,12 \cdot 10^{3} \kappa г, m_{2}=21,1 \cdot 10^{3} \kappa г$ Для динамических характеристик системы будем иметь (таблица 2):

таблица 2

\begin{tabular}{|c|c|c|c|c|c|c|c|c|c|c|}
\hline н/м & н/м & н/м & рад/с & с & рад/с & с & & & & \\
\hline 1,35 & 0,84 & 0,006 & 16,8 & 0,4 & 308,5 & 0,02 & 1 & 365,6 & 1 & $-0,003$ \\
\hline
\end{tabular}

Для значении коэффициента формы определённых согласно выражению

$$
\eta_{i k}=\frac{A_{i k} \sum_{j=1}^{2} Q_{j} A_{i j}}{\sum_{j=1}^{2} Q_{j} A_{i j}^{2}} \quad(i, k=1,2)
$$

Будем иметь (таблица 3)

Таблица 3

\begin{tabular}{|c|c|c|c|c|}
\hline \multirow{2}{*}{} & \multicolumn{2}{|c|}{ Для I формы колебания } & \multicolumn{2}{c|}{ Для II формы колебания } \\
\cline { 2 - 5 } & & & & $-0,0027$ \\
\hline Те же значения масс & 0,0023 & 0,931 & 1 & $-0,003$ \\
\hline Те же значения масс & 0,0027 & 0,987 & 1 & \\
\hline
\end{tabular}

Определяем сейсмические нагрузки в точках сосредоточения масс $m_{1}$ и $m_{2}$ соответственно

$$
S_{i k}=K_{1} K_{2} K_{3} Q_{k} A \beta_{i} K_{0} K_{\psi}(i, k=1,2)
$$

по первой и второй форме колебаний, по формуле (4) при значениях коэффициентов $K_{2}=K_{3}=K_{0}=$ $K_{\psi}=1 ; \quad K_{1}=0,5 ; \quad A=0,17 ; \quad \beta=2,5[1,7]$ и исходя из цели нашей задачи, суммируем сейсмические силы (а не вызванные им усилия) [8] по двум формам колебаний. Окончательно получаем следующие значения сейсмических нагрузок приложенных в точках сосредоточения масс $m_{1}$ и $m_{2}$ :

- для $\left(k / k_{1}\right)=1,25 S_{1}=4,93 \cdot 10^{3}$ н и $S_{2}=$ $43,87 \cdot 10^{3} \mathrm{H}$;

- для $\left(k / k_{1}\right)=1,6 \quad S_{1}=5,08 \cdot 10^{3}$ н и $S_{2}=$ $47,15 \cdot 10^{3} \mathrm{H}$
Сейсмические нагрузки в точке сосредоточения массы определённая по нормативной методике, т.е. как для системы с одной степенью свободы с жёстким защемлением, без наших предположении и для тех же коэффициентов из уравнения (4) будут равняться $S=$ $44,8 \cdot 10^{3} \mathrm{H}$.

\section{Заключение}

Как видим, для соотношения $\left(k / k_{1}\right)=1,25$ значение сейсмических нагрузок в точке сосредоточения массы $m_{2}$ фактически совпадают, а для соотношения $\left(k / k_{1}\right)=1,6$ отличия составляет 5\% от значения, 
полученного по нашей методике. Сейсмическая нагрузка в точке сосредоточения массы незначительна.

Нами рассматривалось только два соотношения для характеризующих параметров системы. В даль- нейшем для рассматриваемого конструктивного решения нами будут получены значения сейсмических нагрузок, полученных на основе статистической обработки полученных результатов для разных соотношении всех характеризующих систему параметров.

\section{Литература}

1. Pn 01.01-9 Construction norms and rules - "Earthquake Engineering". Tb., 2010. (In Georgian).

2. Standard ASCE/SEI 7-10: Minimum design loads for buildings and other structure. 2013, $408 \mathrm{p}$.

3. SP 14.13330.2014. Construction in seismic regions. SNiP - II-7-81 * . M., 2013, 126 p. (in Russian).

4. Iranian code of practice for seismic resistant design of buildings 'Standard 2800". BHRC Publication. N5-465 1st part. 2007, $94 \mathrm{p}$.

5. Eurocode 8: Design of structures for earthquake resistance. Part 1. EN 1998-1. 2004, 231 p.

6. Esadze S., Esadze T. Vertical seismic loads on cantilevers. Proceedings of the 2nd international symposium on seismic stability and engineering. 2019,61 - 65 pp. (in Russian).

7. Birbraer A. Calculation of structures for earthquake resistance. SPb.: "Nauka". 1998, 225 p. (in Russian).

8. Korchinsky I.L. Earthquake resistant building construction. Moscow: "Vysshaya shkola”. 1971. (In Russian). 


\title{
UDC 624.042 \\ SCOPUS CODE 2205
}

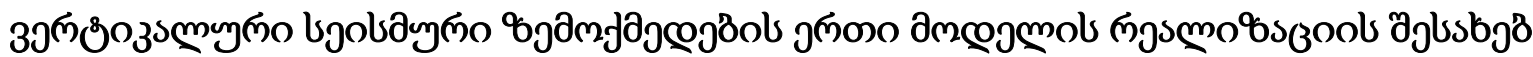

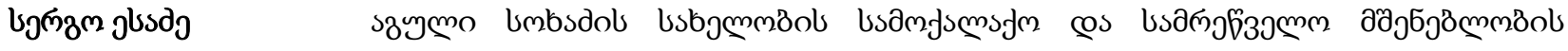

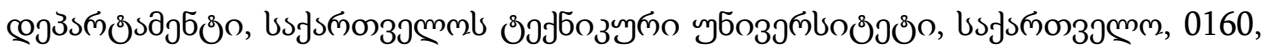

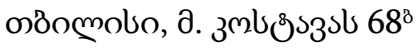 \\ E-mail: s_esadze@gtu.ge

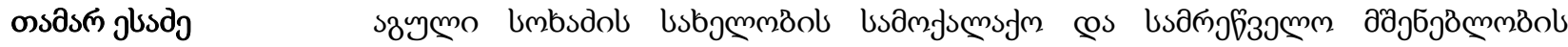

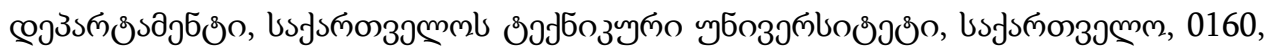

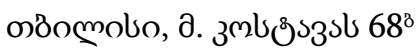 \\ E-mail: t.esadze@gtu.ge

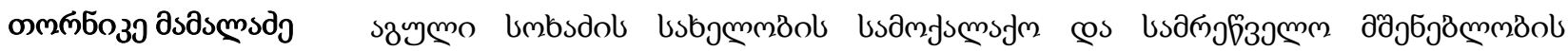

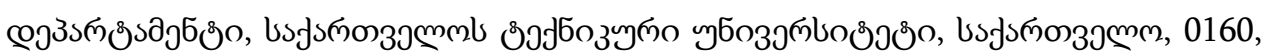

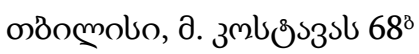 \\ E-mail: tornike.mamaladze1994@gmail.com
}

\section{๓ృ $33^{6 \%}$}

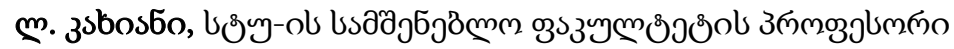

E-mail: L.kakhiani@gtu.ge

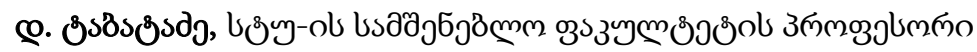

E-mail: Demuritabatadze@gmail.com

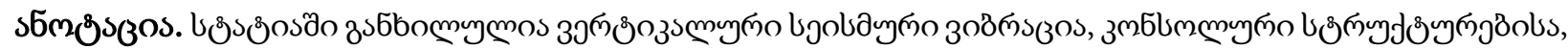

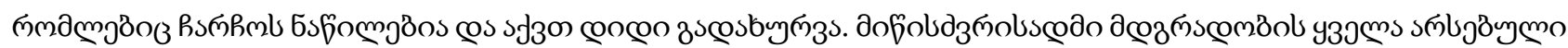

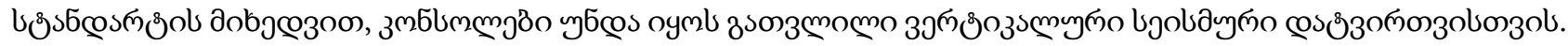

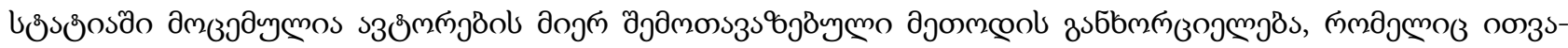

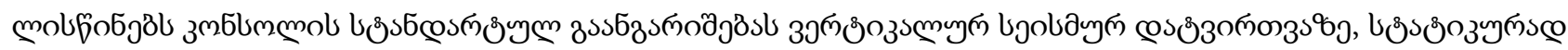

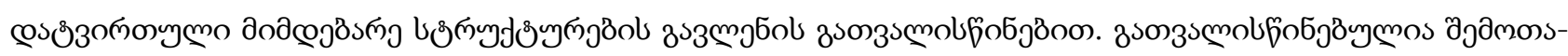
3

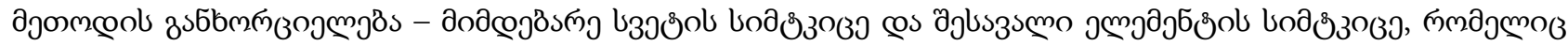

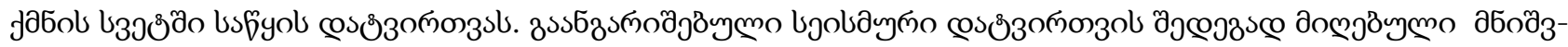

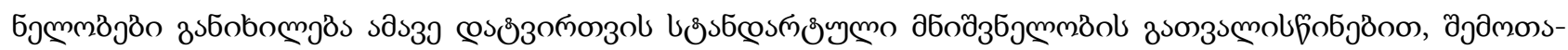

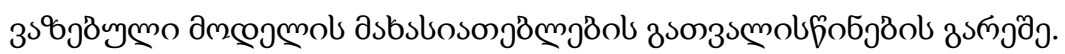

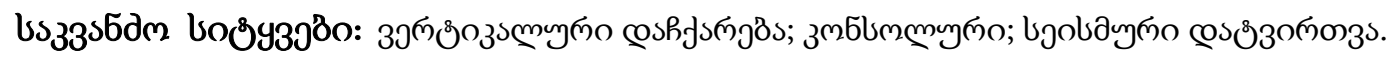




\title{
UDC 6
}

SCOPUS CODE 2205

\section{On the implementation of one model of vertical seismic load}

\author{
Sergo Esadze \\ Aguli Sokhadze Department of Civil and Industrial Engineering, Georgian Technical \\ University, 68 ${ }^{\mathrm{b}}$ M. Kostava str, 0160 Tbilisi, Georgia \\ E-mail: s_esadze@gtu.ge \\ Tamar Esadze Aguli Sokhadze Department of Civil and Industrial Engineering, Georgian Technical \\ University, 68 $\mathrm{b}$ M. Kostava str, 0160 Tbilisi, Georgia \\ M. Kostava str, 0175 Tbilisi, Georgia \\ E-mail: t.esadze@gtu.ge \\ Tornike Mamaladze Aguli Sokhadze Department of Civil and Industrial Engineering, Georgian Technical \\ University, 68 ${ }^{\mathrm{b}}$ M. Kostava str, 0160 Tbilisi, Georgia \\ E-mail: tornike.mamaladze1994@gmail.com
}

\section{Reviewers:}

L. Kakhiani, Professor, Faculty of Civil Engineering, GTU

E-mail: L.kakhiani@gtu.ge

D. Tabatadze, Professor, Faculty of Civil Engineering, GTU

E-mail: Demuritabatadze@gmail.com

\begin{abstract}
The article discusses the vertical seismic vibration of cantilever structures that are part of the frame and have a large overlap. By all existing standards of earthquake resistance, consoles must be designed for vertical seismic loading. The article discusses the implementation of the method proposed by the authors, which provides the standard calculation of the console on the vertical seismic load, taking into account the impact of statistically loaded surrounding structures. It is envisaged to implement two methods of ratio of the characteristic parameters of the proposed calculation model - the strength of the adjacent column and the strength of the input element, which creates the initial load in the column. The values obtained as a result of the calculated seismic load are considered in comparison with the standard value of the same load received without taking into account the characteristics of the proposed model.
\end{abstract}

Key words: Cantilever; seismic load; vertical acceleration.

Дата рассмотрения 24.02.2019

Дата поступления 04.03.2019

Подписано к печати 08.07.2020 